\title{
Antimicrobial activities of Korean mugwort (Artemisia iwayomogi and Artemisia princeps) extracts against Staphylococcus aureus and Cutibacterium acnes
}

\author{
Eun Jeong Park, Jun-Hyun Oh* \\ Department of Plant and Food Sciences, Sangmyung University, Cheonan 31066, Korea
}

\begin{abstract}
Cutibacterium acnes and Staphylococcus aureus are recognized as pus-forming bacteria that trigger skin inflammation in acne. The goal of this research was to determine the antimicrobial activity of Korean mugworts including In-jin-ssuk (Artemisia iwayomogi) and Yak-ssuk (Artemisia princeps). Dried mugwort powders were extracted using water, ethanol and methanol. The antimicrobial activities of the extracts were evaluated by determining minimum inhibitory concentrations (MICs) and squares of inhibition zone widths. The chemical compositions of the chloroform fractions were analyzed by GC/MS. The inhibitory effects of the ethanol and methanol extracts of In-jin-ssuk ( A. iwayomogi) $(20 \mathrm{mg} / \mathrm{mL})$ against $C$. acnes were significantly greater than the inhibitory effect of the water extract $(\mathbf{p}<0.05)$. Among various extracts, the ethanol extract of $A$. iwayomogi exhibited the greatest antimicrobial activity against $S$. aureus. The MICs of the chloroform fractions of ethanol- and methanol-extracted $A$. iwayomogi were determined to be 15 and $10 \mathrm{mg} / \mathrm{mL}$, respectively, against both $C$. acnes and $S$. aureus. The chloroform fraction of the methanol extract exhibited squares of zone widths of 44 and $41 \mathrm{~mm}^{2}$ against $C$. acnes and $S$. aureus, respectively, which were the highest among the tested fractions. Scopoletin $(484 \mathrm{mg} / 100 \mathrm{~g})$ was found to be a major phenolic compound in the chloroform extract, while 2-furanmethanol $(61 \mathrm{mg} / 100 \mathrm{~g})$ and isofraxidin $(55 \mathrm{mg} / 100 \mathrm{~g})$ were minor phenolic compounds.
\end{abstract}

Key words : antimicrobial activity, mugwort, Staphylococcus aureus, Cutibacterium acnes

\section{Introduction}

Acne vulgaris, ordinarily known as acne, is a common skin disease that affects most adolescents and adults at some time in their lives (1). Acne is a chronic inflammatory disease of the pilosebaceous unit. The lesions are localized in "acne prone areas", where sebaceous follicles are most common, which includes the surface skin of the face, neck, chest, and back. Acne generally develops in young people due to several factors including food, stress, the use of cosmetics, hormonal imbalance, or bacterial infection (2). It is accepted that inflammation in acne vulgaris is mainly induced by an immunological response to Cutibacterium acnes (once referred to Propionibacterium acnes) (2). This bacterium

*Corresponding author. E-mail : junhyunoh@smu.ac.kr Phone : 82-41-550-5295; Fax : 82-41-550-5296

Received 11 December 2018; Revised 01 February 2019; Accepted 28 February 2019.

Copyright (c) The Korean Society of Food Preservation. All rights reserved. plays a critical role in the development of inflammatory acne when it overgrows and colonizes the pilosebaceous unit (3).

$C$. acnes is a gram-positive non-spore-forming anaerobic bacterium; it is pleomorphic and rod shaped, and can produce propionic acid. The organism forms units in the normal flora of the oral cavity, large intestine, conjunctiva, external ear canal and skin, where it dominates over other normal flora components in pilosebaceous follicles (4).

Staphylococcus aureus is a common foodborne pathogen capable of causing a variety of human infections (5). In man, $S$. aureus causes two main infection types: (1) cutaneous or mucosal infections and (2) septicemic infections that are generally associated with visceral (abscesses, endocarditis, lung) or bone (osteomyelitis) infections (6). S. aureus has the capacity to readily acquire antimicrobial resistance, which makes remedy difficult. Treatment of acne invloves the use of topical or systemic antibiotics; however, gradual increase in incidences of antibiotic resistance in the causative bacterium has led to a serious need for effective, non-antibiotic treatments (5). 
Many researchers have worked toward the development of remedial agents for acne that do not contribute to resistance but still have high antibacterial activities (1). In recent years, there has been increasing interest in healthy lifestyles and aging using products of plant origin. A large number of aromatic, spicy, medicinal, and other plants that belong to the Asteraceae family contain chemical compounds that exhibit antimicrobial and antioxidant properties (7).

Mugwort (Artemisia) species are part of the Asteraceae family and have been used in traditional medicine to treat serious diseases, including microbial and viral infections (8). Above all, Artemisia iwayomogi Kiramura is a well-known herbal hepatotherapeutic drug that has been traditionally used in Korea and/or China over long periods of time for antitumor, immunomodulating, antimutagenic, antioxidant, antibacterial, antifungal, liver-defense and choleretic purposes $(8,9)$. Artemisia iwayomogi has attracted substantial attention for its potential use as a chemopreventative agent (10). Pharmaceutical researchers have identified a number of terpenoid acids, polyenes, phenols, coumarins, chromones, and flavonoids as the ingredients of the crude drug. There are also numerous reports on the biological activities of Artemisia against human pathogens comprising viruses, fungi, and yeasts (11); however, there are only a few research reports that examine the effects of $A$ iwayomogi against $C$. acnes, one of the major acne-causing bacteria. Therefore, the objectives of this research are 1) to examine the antimicrobial activities of mugworts against $C$. acnes and $S$. aureus, and 2) to identify the major compounds responsible for antimicrobial activity.

\section{Materials and Methods}

\section{Preparation of bacterial cultures}

Cutibacterium acnes KCTC 3314 and Staphylococcus aureus KCTC 3881 were cultured in Reinforced Clostridial Medium (Difco Laboratories, Detroit, MI, USA) and Nutrient broth (Difco Laboratories, Detroit, MI, USA), respectively. The $C$. acnes culture was incubated at $37^{\circ} \mathrm{C}$ for $72 \mathrm{~h}$ under anaerobic conditions in a candle gas jar (GasPak EZ Container Systems, Franklin Lakes, N.J., USA). The $S$. aureus culture was incubated in an incubation shaker at $37^{\circ} \mathrm{C}$ and $200 \mathrm{rpm}$ for $24 \mathrm{~h}$. After incubation, both cultures were washed twice with Butterfield's phosphate buffer (BPB) by centrifuging at $5,000 \mathrm{rpm}$ for $5 \mathrm{~min}$ at $5^{\circ} \mathrm{C}$. The collected bacteria were re-suspended in BPB and the bacterial population was determined by measuring the optical density at $650 \mathrm{~nm}$ with a spectrophotometer (UVmini 1240, Shimadzu, Kyoto, Japan).

\section{Extraction of mugworts}

Korean native mugworts including In-jin-ssuk (Artemisia iwayomogi) and Yak-ssuk (Artemisia princeps) were purchased from a local market located in Daegu, South Korea. The leaves and stems of the mugworts were harvested in the Kangwha area of South Korea during the summer of 2012 and sun-dried immediately after harvesting. Each mugwort was ground into a fine powder prior to extraction. Ground mugwort $(50 \mathrm{~g})$ was separately added to $500 \mathrm{~mL}$ of distilled water, $500 \mathrm{~mL}$ of $80 \%$ ethanol, and $500 \mathrm{~mL}$ of $80 \%$ methanol, and stirred in a water bath at $60^{\circ} \mathrm{C}$ for $12 \mathrm{~h}$. Each extract was then filtered through Whatman No.2 filter paper, and the extracted residue was re-extracted twice (12). The solvent from each extract was completely removed by rotary evaporation. The extracts were freeze-dried and stored at $4{ }^{\circ} \mathrm{C}$ prior to use. The extraction yield of each sample was determined using the following equation: Extraction yield (\%) $=[$ weight of extract $(\mathrm{g}) /$ weight of dried mugwort $(\mathrm{g})] \times 100$.

\section{Fractionation of the organic solvent extracts}

The methanolic or ethanolic mugwort extracts were further fractionated using organic solvents, including $n$-hexane, chloroform, ethyl acetate, and $n$-butanol. $n$-Hexane $(200 \mathrm{~mL})$ and water were added to the alcohol extract in a separating funnel to obtain the $n$-hexane fraction. The $n$-hexane phase was separated, and the remaining aqueous phase was successively treated with various solvents. Chloroform, ethyl acetate and $n$-butanol fractions were obtained using similar procudures (13). The overall fractionation process is illustrated in Fig 1. Following fractionation, each solvent was completely removed by rotary evaporation and each fraction was freeze-dried and stored at $4{ }^{\circ} \mathrm{C}$ prior to further use.

\section{Determination of the antimicrobial activity of mugwort extracts}

The alcoholic mugwort extracts were incorporated into cultural broths at concentrations of $0,5,10,15$, and $20 \mathrm{mg} / \mathrm{mL}$. Streptomycin (5 mg/mL) (Dufhefa, Biochemie, Netherlands) was used as the positive control. Test bacterial cultures were inoculated at concentrations of $10^{6}$ to $10^{8} \mathrm{CFU} / \mathrm{mL}$, and incubated at $37^{\circ} \mathrm{C}$ for $48 \mathrm{~h}$ while shaken at $200 \mathrm{rpm}$. An aliquot of $1 \mathrm{~mL}$ culture was washed twice with BPB by centrifugation at $5,000 \mathrm{rpm}$ for $5 \mathrm{~min}$ using a centrifuge 
(Microcentrifuge 1730R, Labogene, Seoul, Korea). The washed bacteria were re-suspended in $1 \mathrm{~mL}$ of BPB, after which $100 \mu \mathrm{L}$ of the bacterial suspension was transferred in a 96-well microplate (BioTek, Seoul, Korea), and the optical density at $650 \mathrm{~nm}$ was measured using a plate reader (BioTek, Seoul, Korea). The inhibitory effect of the mugwort extract was calculated using the following equation: Inhibitory Effect $(\%)=$ (O.D. measured without the mugwort extract-O.D. measured in the presence of the mugwort extract)/O.D. measured without the mugwort extract $\times 100$.

The $n$-hexane, chloroform, ethyl acetate, and $n$-butanol fractions from the alcoholic mugwort extracts were further examined to determine their minimum inhibitory concentrations (MICs). The broth containing each fraction was prepared as described in the previous section, after which the culture was immediately poured onto Petri plates containing the designated medium for each bacterium. The inoculated plates were incubated at $37^{\circ} \mathrm{C}$ for $24 \mathrm{~h}$ or $72 \mathrm{~h}$. At the end of the incubation period, the plates were evaluated for the presence or absence of growth. The MIC was determined as the lowest concentration of mugwort extract that resulted in the absence of bacterial growth.

The antimicrobial activities of the mugwort extracts were also determined using the agar diffusion method. $C$. acnes and $S$. aureus were cultivated to concentrations of approximately $10^{6} \mathrm{CFU} / \mathrm{mL}$. An aliquot of $1 \mathrm{~mL}$ of each culture was spread onto Reinforced Clostridial agar for $C$. acnes and nutrient agar for $S$. aureus. Blank paper discs of $6 \mathrm{~mm}$ in diameter (BBL, Cockeysville, MD, USA) were impregnated with the specified concentrations of mugwort extracts and placed on the surfaces of the agar plates. The C. acnes plates were then incubated at $37^{\circ} \mathrm{C}$ for $72 \mathrm{~h}$ in an anaerobic jar (GasPak EZ Container Systems, Franklin Lankes, N.J., USA). The $S$. aureus plates were incubated at $37^{\circ} \mathrm{C}$ for $24 \mathrm{~h}$. Streptomycin $(5 \mathrm{mg} / \mathrm{mL})$ and BPS buffer were used as positive and negative controls, respectively. Followng incubation, the diameter of each inhibition zone was measured at two cross-sectional points, and the average was used to calculate the area of the inhibition zone. The antimicrobial activity of each mugwort extract is expressed by the square of zone width according to the following equation (14): Square of zone width $\left(\mathrm{mm}^{2}\right)=$ [diameter of clear zone-diameter of the blank disc $(6 \mathrm{~mm})] / 2$.

\section{Identification of mugwort extracts by GC/MS}

Mugwort samples were extracted using $80 \%$ methanol and filtered through filter paper. The methanol was completely removed from the extract by rotary evaporation. Each residual extract was dissolved in water for further fractionation with chloroform. GC/MS was performed using a 6,890 GC gas chromatograph coupled to a 5,975 mass spectrometer (HP, Palo Alto, CA, USA). Compounds were separated on a 30 $\mathrm{m} \times 0.25 \mathrm{~mm}$ i.d. capillary column with a 0.25 - $\mu \mathrm{m}$-thick coating (15). The column temperature was maintained at $60^{\circ} \mathrm{C}$ for $5 \mathrm{~min}$ after injection, then programmed to increase at $10^{\circ} \mathrm{C} / \mathrm{min}$ to $250^{\circ} \mathrm{C}$, and maintained at that temperature for $5 \mathrm{~min}$. Split injection with a split ratio of 5:1 was used, and helium was used as the carrier gas at a flow rate of $1 \mathrm{~mL} / \mathrm{min}$. The spectrometer was operated over the 29-800 amu mass scan range, and a solvent delay time of $3 \mathrm{~min}$ was employed. The chemical constituents were identified by comparing their relative retention times and mass spectra with those obtained from authentic sample and/or the NIST/NBS and Wiley libraries spectra (9).

\section{Statistical analysis}

Each experiment was repeated three times. One way analysis of variance (ANOVA) was performed using SPSS software (version 11.5, SPSS Inc., Chicago, IL, USA). Differences among means were analyzed using Duncan's Multiple Range test (DMRT), and the significance level was defined as $\mathrm{p}<0.05$.

\section{Results and Discussion}

\section{Inhibitory effects of mugwort extracts against acne-causing bacteria}

The extraction yields of Yak-ssuk ( $A$ princeps) using water, ethanol, and methanol were 13.9, 9.1, and 10.8\%, respectively. Similar extraction yields were also obtained for In-jin-ssuk ( $A$ iwayomogi). The highest yield was obtained from the water extract, whereas the lowest yield was obtained from the methanol extract. Kim et al. (16) also reported that the highest extraction yield was obtained from the water extract of Hizikia fusiforme. These results indicate that the variances in extraction yields among the selected solvents are attributed to differences in solvent polarity, which results in different compounds extracted from the mugworts by the various solvents.

The inhibitory effects of mugwort extracts against $C$. acnes and $S$. aureus are presented in Figs. 2 and 3, respectively. The inhibitory effects of the In-jin-ssuk ( $A$ iwayomogi) and Yak-ssuk ( $A$ princeps) extracts were both observed to 


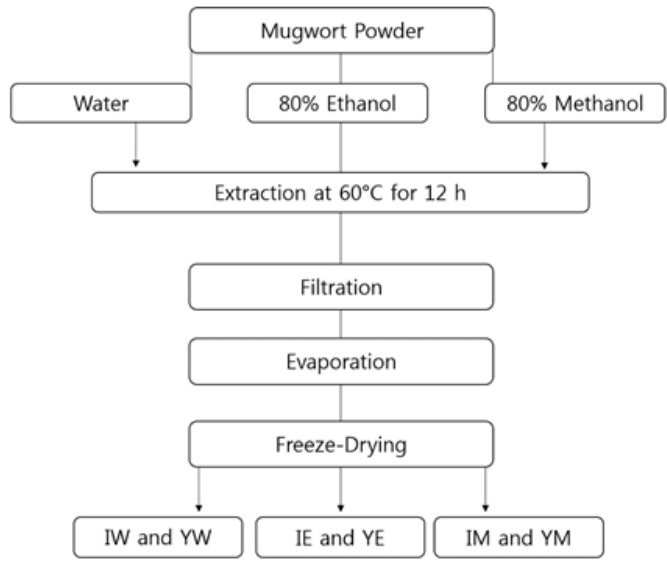

(A)

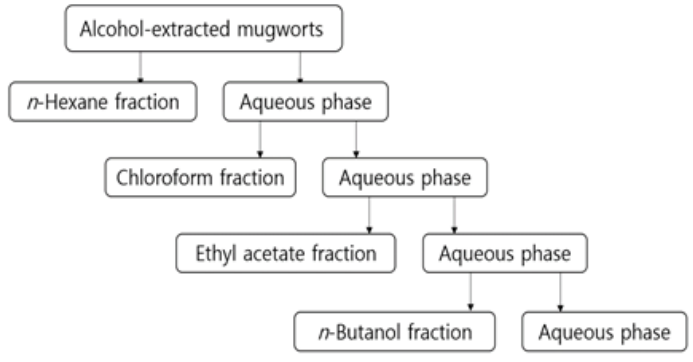

(B)

Fig. 1. Overall sequence of solvent extraction (A) and fractionation (B) of mugworts.

IW : In-jin-ssuk water extract, YW : Yak-ssuk water extract, IE : In-jin-ssuk ethanol extract, YE : Yak-ssuk ethanol extract, IM : In-jin-ssuk methanol extract, YM : Yak-ssuk methanol extract

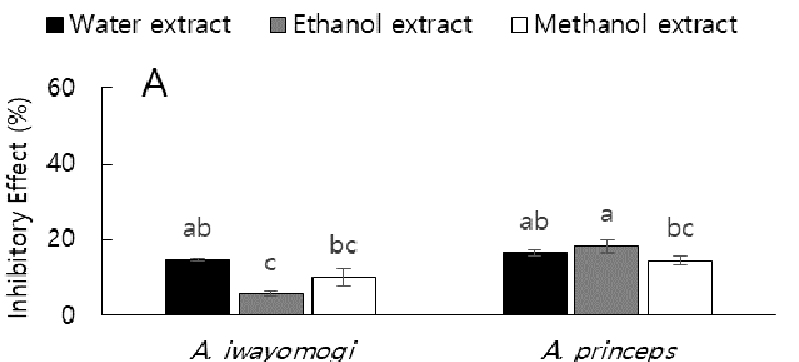

Water extract

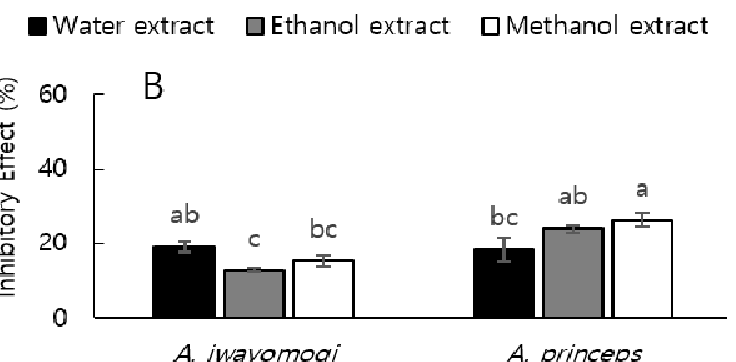

Water extract $\square$ Ethanol extract $\square$ Methanol extract

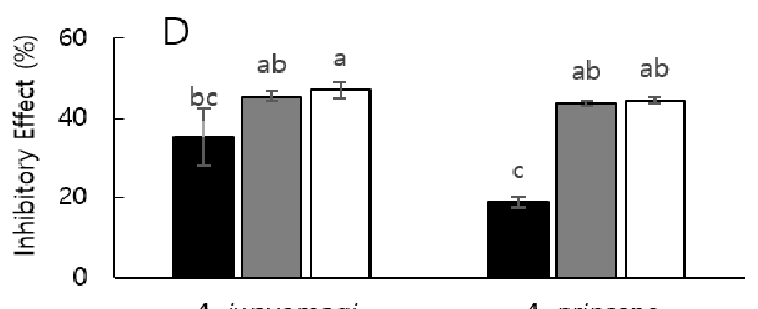

A. princeps

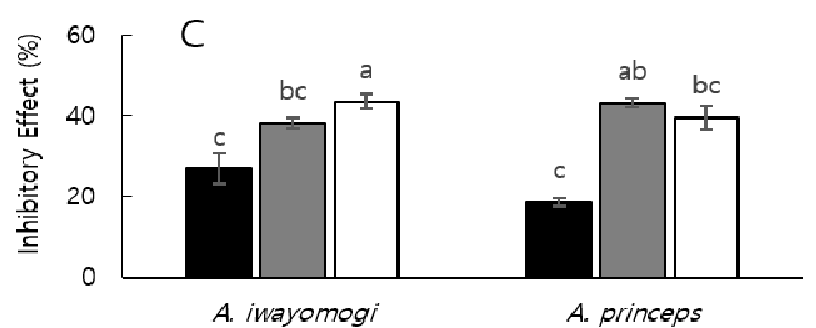

A. iwayomogi

Fig. 2. Growth inhibitory effects of different concentrations of the various extracts from In-jin-ssuk (Artemisia iwayomogi) and Yak-ssuk (Artemisia princeps) against Cutibacterium acnes.

A, concentration of extracts $(5 \mathrm{mg} / \mathrm{mL})$; B, concentration of extracts $(10 \mathrm{mg} / \mathrm{mL})$; C, concentration of extracts $(15 \mathrm{mg} / \mathrm{mL})$; D, concentration of extracts $(20 \mathrm{mg} / \mathrm{mL})$. Error bars represent standard deviation $(n=3)$. Different letters $(\mathrm{a}-\mathrm{c})$ represent significant differences among the samples at $\mathrm{p}<0.05$. Inhibitory Effect $(\%)$ was calculated by the following equation; (O.D. at $650 \mathrm{~nm}$ measured without mugwort extract-O.D. at $650 \mathrm{~nm}$ value measured in the presence of mugwort)/O.D. at $650 \mathrm{~nm}$ value measured without mugwort extract.

increase as the extract concentrations increased from 5 to $20 \mathrm{mg} / \mathrm{mL}$. At $20 \mathrm{mg} / \mathrm{mL}$, the inhibitory effects of the ethanol and methanol extracts of the two mugworts against $C$. acnes were significantly higher than those of the water extracts $(p<0.05)$; however there was no significant difference between the ethanol and methanol extracts (Fig. 2D).
The mugwort extracts exhibited similar trends toward $S$. aureus to those observed for $C$. acnes, however a significant difference was observed between the two alcohol extracts. The ethanol extract from In-jin-ssuk exhibited the highest inhibitory effects, while the methanol extract from Yak-ssuk was most active (Fig. 3D). These results reveal that alcohols, 
such as ethanol or methanol, are more effective solvents for extracting antimicrobial compounds present in mugwort than water; therefore, the ethanol and methanol mugwort extracts were further fractionated.

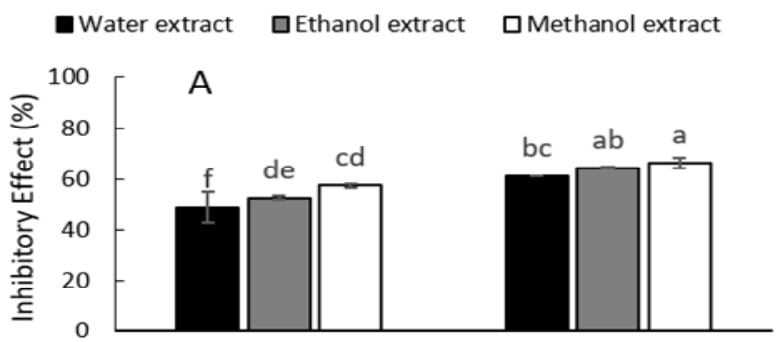

A. iwayomogi

A. princeps

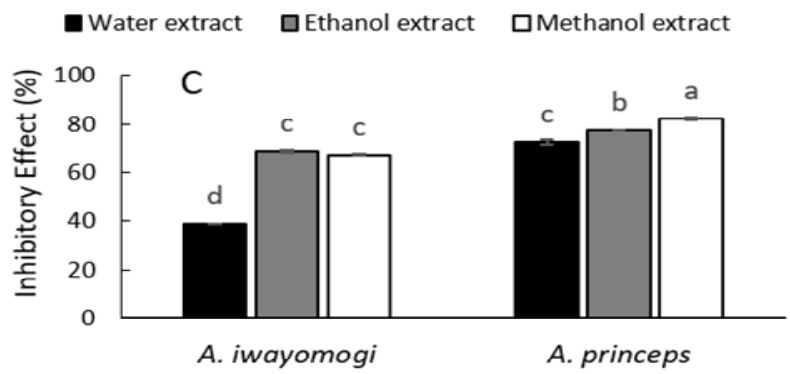

Fig. 3. Growth inhibitory effects of different concentrations of the various extracts from In-jin-ssuk (Artemisia iwayomogi) and Yak-ssuk (Artemisia princeps) against Staphylococcus aureus.

A, concentration of extracts $(5 \mathrm{mg} / \mathrm{mL}) ; \mathrm{B}$, concentration of extracts $(10 \mathrm{mg} / \mathrm{mL}) ; \mathrm{C}$, concentration of extracts $(15 \mathrm{mg} / \mathrm{mL}) ; \mathrm{D}$, concentration of extracts $(20 \mathrm{mg} / \mathrm{mL})$. Error bars represent standard deviation $(n=3)$. Different letters $(a-c)$ represent significant differences among the samples at $p<0.05$. Inhibitory Effect $(\%)$ was calculated by the following equation; (O.D. at $650 \mathrm{~nm}$ measured without mugwort extract-O.D. at $650 \mathrm{~nm}$ value measured in the presence of mugwort)/0.D. at $650 \mathrm{~nm}$ value measured without mugwort extract.

Minimum inhibitory concentration of the $A$. iwayomogi extract

The antimicrobial activities of both the ethanol and methanol extracts of In-jin-ssuk ( $A$ iwayomogi) fractionated with selected solvents were further evaluated by determining their MICs, which are the lowest concentrations of the antimicrobials that inhibit the visible growth of a microorganism. Antimicrobial potency was qualitatively and quantitatively assessed using the MIC values. As presented in Table 1, fractionation of the alcohol extracts of In-jin-ssuk ( $A$ iwayomogi) using selected solvents led to improved antimicrobial activities against acne-causing bacteria. The MIC of the chloroform fraction of the methanol extract was found to be $10 \mathrm{mg} / \mathrm{mL}$ against both $C$. acnes and $S$. aureus, while the MIC of the chloroform fraction of the ethanol extract was $15 \mathrm{mg} / \mathrm{mL}$ against both bacterial strains.

The antimicrobial activities (MICs) of the $n$-hexane and ethyl acetate fractions were found to be $20 \mathrm{mg} / \mathrm{mL}$ against

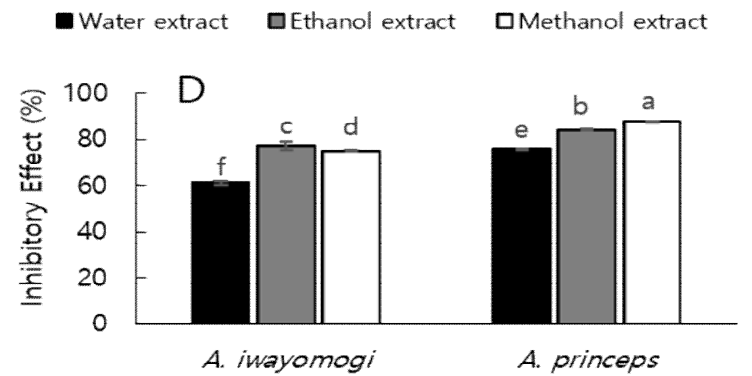

both $C$. acnes and $S$. aureus. The MICs of the ethyl acetate and hexane soluble extracts of Eisenia bicyclis have previously been reported to be $64 \mu \mathrm{g} / \mathrm{mL}$ and $128 \mu \mathrm{g} / \mathrm{mL}$, respectively, against $C$. acnes (17). The MICs of tea-tree oil



were determined to be between $0.31 \%$ and $0.63 \%$ for $C$. acnes, and between $0.63 \%$ and $1.25 \%$ for $S$. aureus, indicating that tea-tree oil possess substantial antimicrobial activities against both $C$. acnes and $S$. aureus (18). Extracts of rose, duzhong, and yerba mate also exhibit notable antimicrobial activities against $C$. acnes (19). Among these extracts, the duzhong extract is antimicrobially most active against $C$. acnes with an MIC of $0.5 \mathrm{mg} / \mathrm{mL}$, while the yerba mate extract showed moderate antibacterial activity against $C$. acnes with an MIC of $1 \mathrm{mg} / \mathrm{mL}$. In addition, the in vitro antimicrobial activities of more essential oils, medicinal plants, and chemicals against $C$. acnes have been reported by several research groups (20-22).

Antimicrobial activities of the solvent fractions of alcohol extracts

The antimicrobial potencies of the mugwort extracts were qualitatively and quantitatively assessed by the presence or 
Table 1. Minimum inhibitory concentrations of fractionated $A$ iwayomogi extracts against acne-causing bacteria

\begin{tabular}{ccccccc}
\hline \multirow{2}{*}{ Bacteria } & \multirow{2}{*}{ Extract } & \multicolumn{5}{c}{ Minimum inhibitory concentration (mg/mL) } \\
\cline { 3 - 6 } & & $n$-Hexane & Chloroform & Ethyl & acetate & $n$-Butanol \\
\hline Cutibacterium & Ethanol & 20 & 15 & 20 & $>20$ & $>20$ \\
acnes & Methanol & 20 & 10 & 20 & $>20$ & $>20$ \\
\hline Staphylococcus & Ethanol & 20 & 15 & 20 & \\
aureus & Methanol & 20 & 10 & & 20 \\
\hline
\end{tabular}

absence of inhibition zones, and by the diameters of these zones. Although the alcohol extracts of In-jin-ssuk $(A$ iwayomogi) inhibited the growth of both $C$. acnes and $S$. aureus, these extracts did not produce distinct clear zones when tested using the disc diffusion method. Further fractionation of the alcohol extracts of In-jin-ssuk ( $A$ iwayomogi) using selected solvents resulted in extracts with higher antimicrobial activities against both $C$. acnes and $S$. aureus, these extracts produced measurable clear zones in the disc diffusion assay. The clear zones of the solvent-fractionated In-jin-ssuk ( $A$ iwayomogi) from the methanol extracts are presented in Fig. 4. Among the fractions, the chloroform fractions of In-jin-ssuk ( $A$ iwayomogi) exhibited the largest

clear zones against both $C$. acnes and $S$. aureus, when tested at concentration of $20 \mathrm{mg} / \mathrm{mL}$. The square of zone width of a clear zone is often used to relate the antimicrobial activity of a compound (14). As presented in Fig. 5, the chloroform fractions from the methanol and ethanol extracts were calculated to have the largest squares of zone widths among the fractions, while the $n$-butanol fractions from the methanol and ethanol extracts exhibited the smallest squares of zone widths. Tea-tree oil at a concentration of $5 \mathrm{~mL}$ has been reported to show squares of zone widths of $78 \mathrm{~mm}^{2}$ and $230 \mathrm{~mm}^{2}$ for inhibition zones against $C$. acnes and $S$. aureus, respectively (18). The squares zone widths of the clear zones from the In-jin-ssuk ( $A$. iwayomogi) fractions against $S$. aureus exhibited similar trends to those observed for $C$. acnes (Fig. 6). The results of a previous study showed similar results to those of the chloroform fractions of the In-jin-ssuk ( $A$ iwayomogi) extract in this study.

The clear-zone size of the chloroform fraction of the methanol extracted In-jin-ssuk ( $A$ iwayomogi) was observed to increase with increasing fraction concentration (Fig. 7). The largest inhibition zone was observed at a chloroform-fraction concentration of $20 \mathrm{mg} / \mathrm{mL}$ (Fig. 8). Based on these results, the chloroform fractions from methanol extracts exhibit the strongest antimicrobial activities

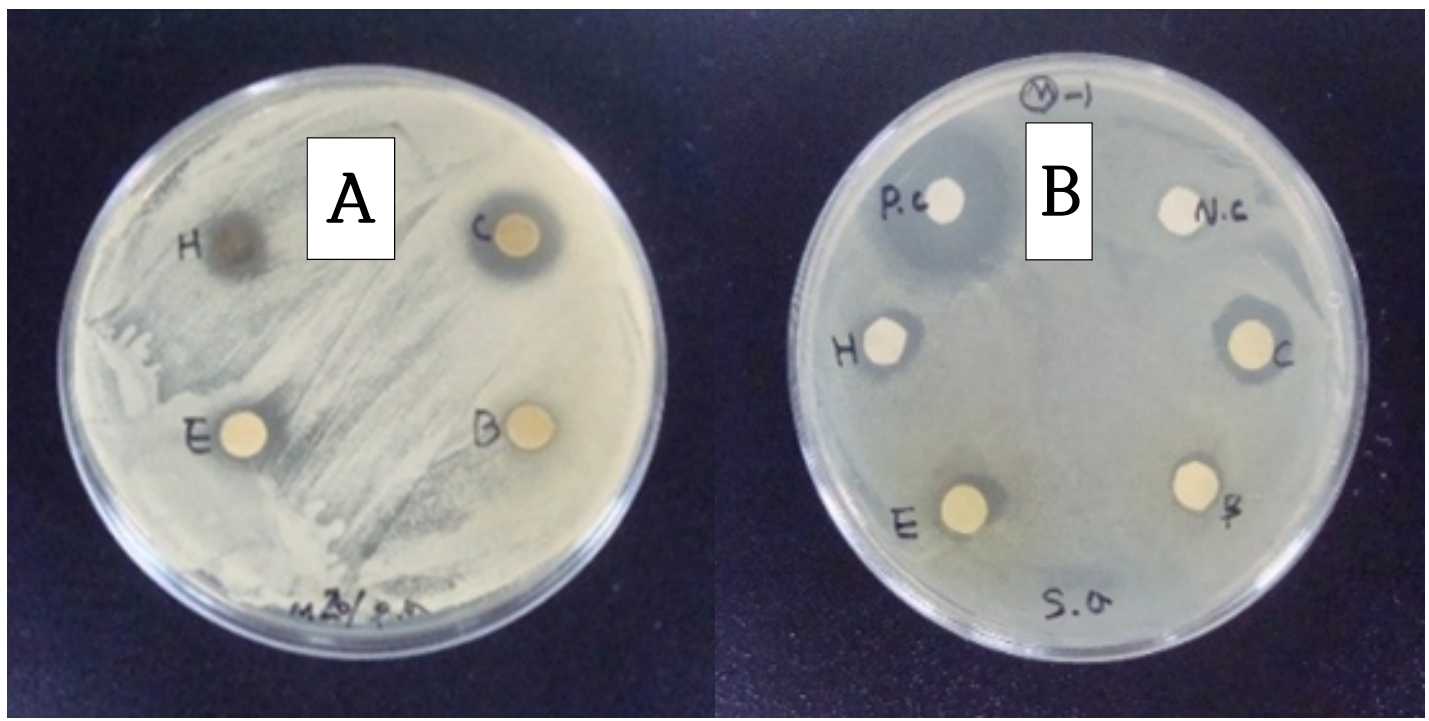

Fig. 4. Clear zones of $A$ iwayomogi extracts fractionated using selected solvents from the methanol extract against (A) Cutibacterium acnes and (B) Staphylococcus aureus.

Symbols: N.C., negative control with DMSO; P.C., positive control with streptomycin $(5 \mathrm{mg} / \mathrm{mL}) ; \mathrm{H}, \mathrm{n}$-hexane fraction $(20 \mathrm{mg} / \mathrm{mL}) ; \mathrm{C}$, chloroform fraction $(20 \mathrm{mg} / \mathrm{mL}) ; \mathrm{E}$, ethyl acetate fraction $(20 \mathrm{mg} / \mathrm{mL})$; and $\mathrm{B}$, n-butanol fraction $(20 \mathrm{mg} / \mathrm{mL})$. 


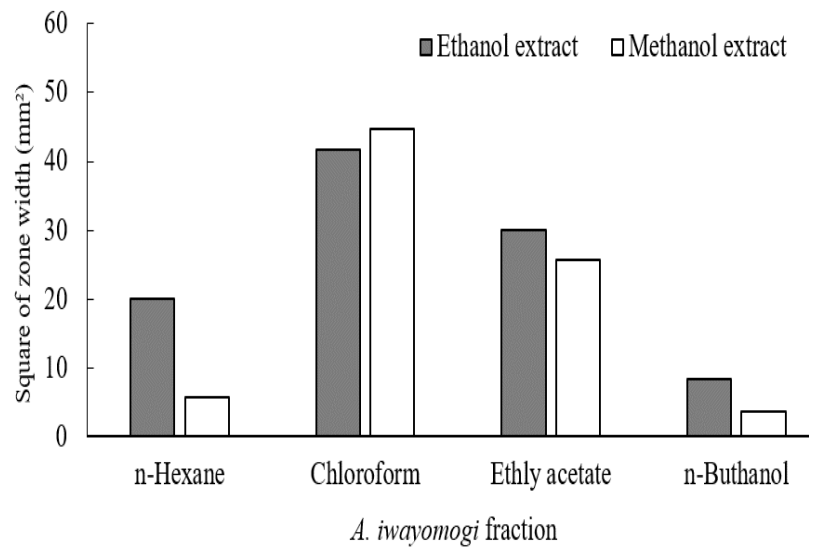

Fig. 5. Square zone widths of clear zones of $A$ iwayomogi fractions using selected solvents from the alcohol extracts against Cutibacterium acnes determined by disc diffusion method $(\mathrm{p}<0.05)$.

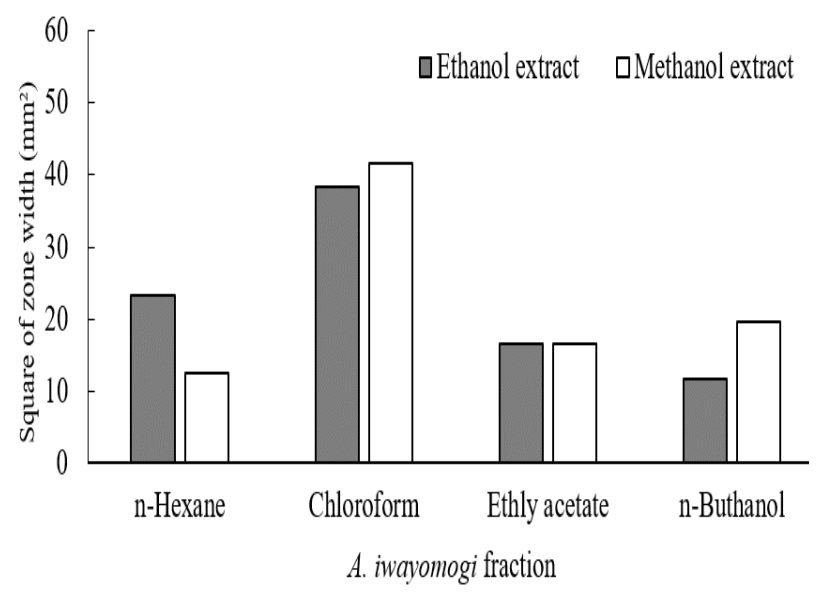

Fig. 6. Square zone widths of clear zones of $A$ iwayomogi fractions using selected solvents from the alcohol extracts against Staphylococcus aureus determined by disc diffusion method $(\mathrm{p}<0.05)$. against $C$. acnes among the tested fractions with the lowest MIC of $10 \mathrm{mg} / \mathrm{mL}$ and the largest inhibition zone (12-18 $\mathrm{mm}$ diameter). Therefore, the chloroform fraction from the methanol extract of In-jin-ssuk ( $A$ iwayomogi) was further analyzed by GC/MS in order to identify the compounds responsible for antimicrobial activity.

Identification of polyphenolic compounds in the $A$. iwayomogi extracts by $\mathrm{GC} / \mathrm{MS}$

The GC/MS trace of the chloroform fraction of the In-jin-ssuk ( $A$ iwayomogi) methanol extract is presented in Fig. 9, which reveals that scopoletin (48.39\%), isofraxidin $(5.49 \%)$ and 2 -furanmethanol $(6.12 \%)$ are main components of the In-jin-ssuk ( $A$ iwayomogi) extract (Table 2 ). This is in agreement with literature reports for Artemisia species (7). Scopoletin and isofraxidin, which are phenolic compounds, belong to the sesquiterpene lactone family (23). It is believed that scopoletin, the major phenolic constituent in the In-jin-ssuk ( $A$ iwayomogi) extract, contributes to the antimicrobial and antioxidant activities of mugwort. 2-Furanmethanol or furfuryl alcohol is produced during various sugar/amino acid browning reactions $(24,25)$, and various furan derivatives, including 2-furanmethanol, exhibit antioxidant activities $(26,27)$.

Most polyphenolic compounds that exhibit antimicrobial activities are phenolic acids or flavonoids. Phenolic acids form a major class of phenolic compound found in a variety of plants (7), and the phenolic moiety plays a significant role in determining the antimicrobial and antioxidant activities of the plant (28). Mixtures of phenolic acids and other organic compounds can exhibit inhibitory effects even when the
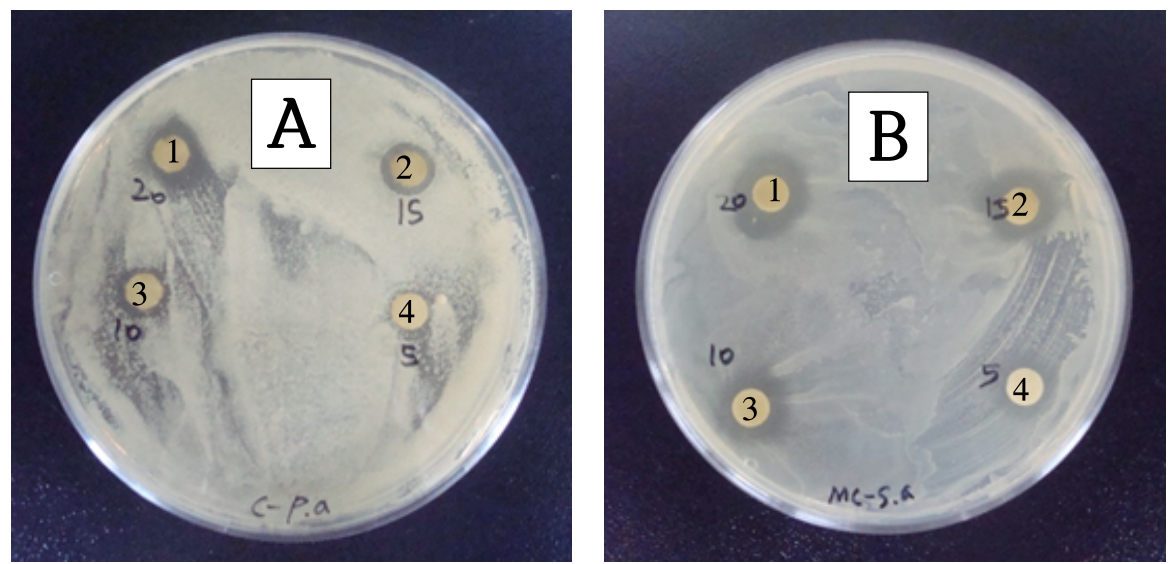

Fig. 7. Inhibitory effects of different concentrations of the chloroform extracts from the methanol extracts of In-jin-ssuk against pathogens. A, Cutibacterium acnes; B, Staphylococcus aureus.

1, $20 \mathrm{mg} / \mathrm{mL} ; 2,15 \mathrm{mg} / \mathrm{mL} ; 3,10 \mathrm{mg} / \mathrm{mL} ; 4,5 \mathrm{mg} / \mathrm{mL}$. 


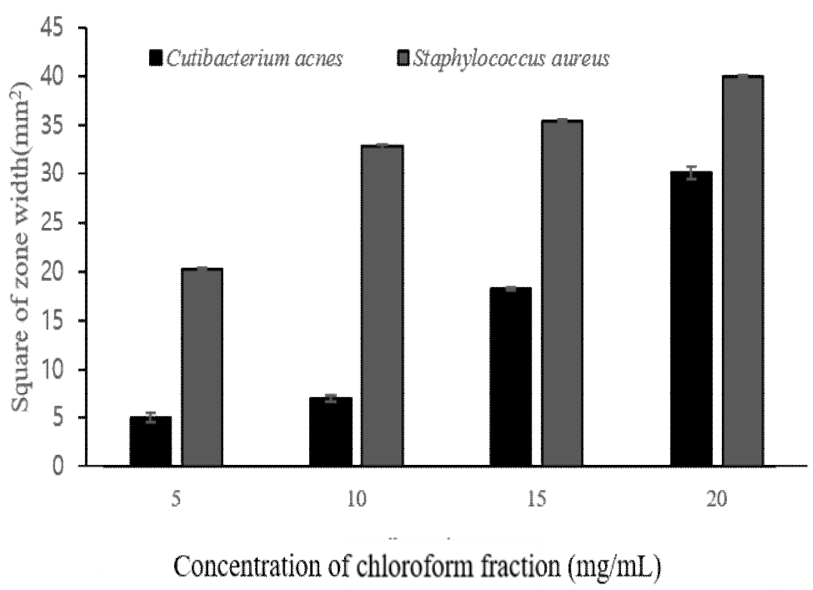

Fig. 8. Square zone widths of clear zones of the $A$ iwayomogi fractions using selected concentrations of chloroform from the alcohol extracts against Cutibacterium acnes and Staphylococcus aureus determined by disc diffusion method $(\mathrm{p}<0.05)$. concentrations of the compounds are below their individual inhibitory levels (29). Hence, the antimicrobial compounds in In-jin-ssuk ( $A$ iwayomogi) are potentially useful as safe and eco-friendly bactericides.

\section{Conflict of interest}

The authors have no conflict of interest.

\section{Acknowledgement}

This research was supported by a 2017 Research Grant from Sangmyung University.

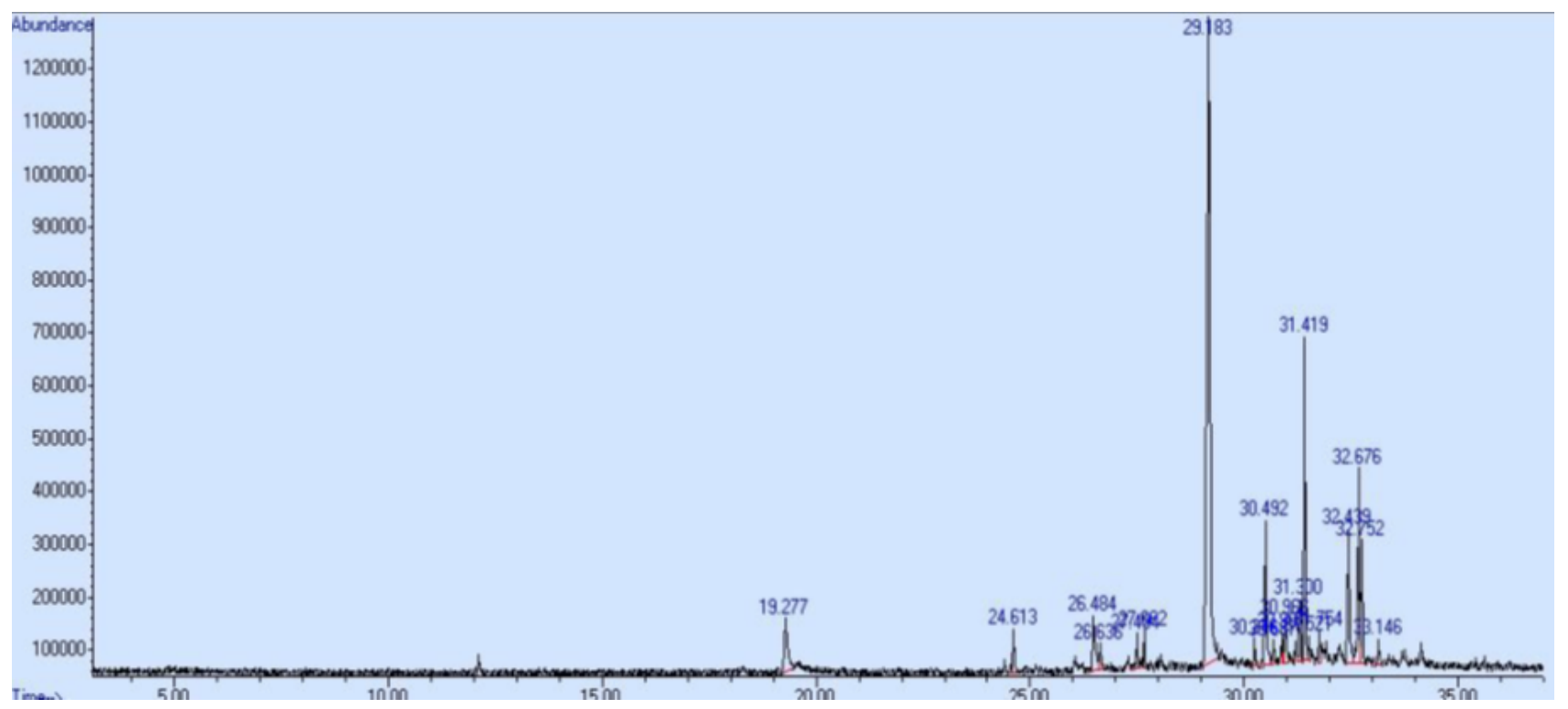

Fig. 9. Gas chromatography/mass spectrometric chromatogram of the the chloroform fraction of the $A$ iwayomogi extract.

Table 2. Chemical composition of the chloroform fraction of the $A$ iwayomogi extracts analyzed with GC/MS

\begin{tabular}{ccccc}
\hline No. & Retention time (min) & Compound name & \multicolumn{2}{c}{ Relative content } \\
$(\%)$ & 48.39 \\
1 & 29.18 & Scopoletin & 5.49 & 8.85 \\
2 & 30.48 & Isofrxidin & 6.12 \\
3 & 31.41 & 1,3,5-Trimethyl benzene & 6.34 \\
4 & 32.43 & 2-Furanmethanol & 3.94 \\
5 & 32.67 & 2,3-Dimethyl-5,6-dimethyllidene-7-oxabicyclo[3.3.2]hept-2-ene & \\
6 & 32.75 & $5,6,7,8-$ Tetrahydro-1-maphthalenol & 20.87 \\
\end{tabular}




\section{References}

1. Bae HJ (2008) Screening of seaweed extracts for antimicrobial activity against Propionibacterium acnes and Staphylococcus epidermidis, which cause acne vulgaris. MS Thesis, Shilla University, Korea, p 23-30

2. Koreck A, Pivarcsi A, Dobozy A, Kemeny L (2003) The role of innate immunity in the pathogenesis of acne. Dermatology, 206, 96-105

3. Nakatsuji T, Kao MC, Fang JY, Zouboulis CC, Zhang L, Gallo RL, Huang CM (2009) Antimicrobial property of lauric acid against Propionibacterium acnes. its therapeutic potential for inflammatory acne vulgaris. J Invest Dermatol, 129, 2480-2488

4. Perry AL, Lambert PA (2006) Propionibacterium acnes. J Appl Microbiol, 42, 185-188

5. Nagaraju U, Bhat G, Kuruvila M, Pai GS, Jayalakshmi, Babu RP (2004) Methicillin-resistant Staphylococcus aureus in community acquired pyoderma. Int J Dermatol, 43, 412-414

6. Sutra L, Poutrel B (1994) Virulence factors involved in the pathogenesis of bovine inflammatory infections due to Staphylococcus aureus. J Med Microbiol, 40, 79-89

7. Seo KS, Jeong HJ, Yun KW (2010) Antimicrobial activity and chemical components of two plants, Artemisia capillaris and Artemisia iwayomogi, used as Korean herbal InJin. J Ecol Environ, 33, 141-147

8. Park Y, Noh HJ, Han L, Kim HS, Kim YJ, Choi JS, Kim CK, Kim YS, Cho S (2012) Artemisia capillaris extracts as a green factory for the synthesis of silver nanoparticles with antibacterial activities. J Nanosci Nanotechnol, 12, 7087-7095

9. Cha JD (2007) Chemical composition and antibacterial activity aginst oral bacteria by essential oil of Artemisia iwayomogi. J Bacteriol Virol, 37, 129-136

10. Wang JH, Choi MK, Shin JW, Hwang SY, Son CG (2012) Antifibrotic effects of Artemisia capillaris and Artemisia iwayomogi in a carbon tetrachloride-induced chronic hepatic fibrosis animal model. J Ethnopharmacol, 140, 179-185

11. Tan RX, Zheng WF, Tang HQ (1998) Biologically active substances from the genus Artemisia. Planta Med, 64, 295-302

12. Liang B (2012) Antibacterial activities of Clitocybe nuda extract on foodborne pathogens. MS Thesis, Auburn University, USA, p 43-51

13. Harborne AJ (1998) Phenolic compounds. In:
Phytochemical Methods: A Guide to Modern Techniques of Plant Analysis, Harborne AJ (Editor), Chapman \& Hall, Springer, London. UK, p 40-106

14. Hewitt W, Vincent S (1989) Theory and application of microbiological assay. Academic Press, San Diego, USA, p 20-30

15. Guo FQ, Liang YZ, Xu CJ, Li XN, Huang LF (2004) Analyzing of the volatile chemical constituents in Artemisia capillaris herba GC-MS and correlative chemometric resolution methods. J Pharm Biomed Anal, $35,469-478$

16. Kim SH, Lim SB, Ko YH, Oh CK, Oh MC, Park CS (1994) Extraction Yields of Hizikia fusiforme by solvents and their antimicrobial effects. Korean J Fish Aquat Sci, 27, 462-468

17. Lee EH (2014) Anti-bacterial activity and antiinflammatory activity of the phlorotannins from Eisenia bicyclis against Propionibacterium acnes. MS Thesis, Pukyong National University, Korea, p 35-50

18. Raman A, Weir U, Bloomfield SF (1995) Antimicrobial effects of tee-tree oil and its major components on Staphylococcus aureus, staph. Epodermidis and Propionibacterium acnes. Lett Appl Microbiol, 21, 242-245

19. Tsaia TH, Tsaib TH, Wu WH, Tsenga JTP, Tsai PJ (2010) In vitro antimicrobial and anti-inflammatory effects of herbs against Propionibacterium acnes. Food Chem, 119, 964-968

20. Chomnawang MT, Surassmo S, Nukoolkarn VS, Gritsanapan W (2005) Antimicrobial effects of Thai medicinal plants against acne-inducing bacteria. J Ethnophamacol, 101, 330-333

21. Docherty JJ, Mcewen HA, Sweet TJ, Bailey E, Booth TD (2007) Resveratrol inhibition of Propionibacterium acnes. J Antimicrob Chemother, 59, 1182-1184

22. Kim SS, Baik JS, Oh TH, Yoon WJ, Lee NH, Hyun CG (2008) Biological activities of Korean citrus obovoides and citrus natsudaidai essential oils against acne-inducing bacteria. Biosci Biotechnol Biochem, 72, 2507-2513

23. Lin F, Hasegawa M, Kodama O (2003) Purification and identification of antimicrobial sesquiterpene lactones from yacon (Smallanthus sonchifolius) leaves. Biosci Biotech Biochem, 67, 2154-2159

24. Shibamoto T (1983) Heterocyclic compounds in browning and browning/nitrite model systems: occurrence, formation mechanisms, flavor characteristics and 
mutagenic activity. In: Instrumental Analysis of Foods: Recent Progress volume 1, Charalambous G, Inglett G (Editors), Academic Press, New York, USA, p 229-278

25. Ames JM, Guy RCE, Kipping GJ (2001) Effect of pH and temperature on the formation of volatile compounds in cysteine/reducing sugar/starch mixtures during extrusion cooking. J Agric Food Chem, 49, 1885-1894

26. Fuster MD, Mitchell AE, Ochi H, Shibamoto T (2000) Antioxidative activities of heterocyclic compounds formed in brewed coffee. J Agric Food Chem, 48, $5600-5603$
27. Yanagimoto K, Lee KG, Ochi H, Shibamoto T (2002) Antioxidative activity of heterocyclic compounds found in coffee volatiles produced by Maillard reaction. J Agric Food Chem, 50, 5480-5484

28. Kujumgiev A, Bankova V, Ignatova A, Popov S (1993) Antibacterial activity of propolis, some of its components and their analogs. Pharmazie, 48, 785-786

29. Blum U (1996) Allelopathic interactions involving phenolic acids. J Nematol, 28, 259-267 